\title{
Métodos de detección de la violencia de género en urgencias por el personal de enfermería
}

\author{
Sara Almenar Campos \\ salmenarcampos@gmail.com \\ Pablo Salas Medina \\ psalas@uji.es
}

\section{Resumen}

Introducción: La violencia de género es una forma de discriminación hacia las mujeres y designa todos los actos de violencia basados en el género que implican daños o sufrimientos de naturaleza física, sexual, psicológica o económica. La mujer maltratada puede acudir a los servicios de urgencias hospitalarias ante la necesidad de atención urgente, siendo estos un punto de detección de esta violencia.

Objetivos: Dar a conocer los métodos de detección disponibles para identificar la violencia de género en el servicio de urgencias.

Metodología: Se realizó una revisión integradora de carácter descriptivo de los artículos originales relacionados con los métodos de detección de la violencia de género en urgencias. La búsqueda bibliográfica se llevó a cabo en las bases de datos a través de las páginas de PubMed, Cinahl, la Biblioteca Cochrane Plus y la Biblioteca Virtual de la Salud.

Resultados: Se obtuvieron un total de 125 artículos. Se seleccionaron y estudiaron finalmente un total de 11 artículos que cumplían con los criterios de selección establecidos. De la base de datos de PubMed se seleccionaron $36,36 \%(n=4)$ artículos; de la base de datos de la Biblioteca Virtual de la Salud no se incluyó ningún artículo (0\%); se extrajo de la Biblioteca Cochrane un $9,09 \%(n=1)$ y un $54,54 \%(n=6)$ artículos se obtuvieron de la base de datos de Cinahl.

Conclusiones: Los servicios de urgencias son un lugar privilegiado para detectar e intervenir en casos de violencia de género. En esta revisión se recogen varias intervenciones de detección de la violencia de género.

Palabras clave: violencia de género, violencia doméstica, enfermería, servicios médicos de urgencia.

\section{Abstract}

Introduction: Gender-based violence is a form of discrimination against women, and it is defined as any act of gender-based violence that involves 
damage or suffering of a physical, sexual, psychological or economic nature. The woman who has been ill-treated can go to the hospital emergency services when faced with the need for urgent attention, these being a point of detection of this violence.

Objectives: To make known the detection methods available to identify gender-based violence in the emergency department.

Methodology: An integrative descriptive review was carried out of the original articles related to methods for detecting gender violence in the emergency department. The bibliographic search was carried out in the databases through the pages of PubMed, Cinahl, the Cochrane Plus Library and the Virtual Health Library.

Results: A total of 125 articles were obtained. A number of 11 articles were selected and finally studied, satisfying the established selection criteria. $36.36 \%(n=4)$ articles were selected from the PubMed database; no articles were included from the Biblioteca Virtual de la Salud database (0\%); $9.09 \%$ $(n=1)$ were extracted from the Cochrane Library; and $54.54 \%(n=6)$ articles were obtained from the Cinahl database.

Conclusions: Emergency services are a privileged place to detect and intervene in cases of gender-based violence. This review includes several interventions for the detection of gender-based violence.

Keywords: gender-based violence, domestic violence, nursing, emergency medical services.

\section{Introducción}

En la sociedad actual, la discriminación sufrida por las mujeres y la violencia de género es un problema que traspasa fronteras y está presente en todos los países del mundo (Xunta de Galicia s.f.). La base de este tipo de violencia se encuentra en la discriminación que sufren las mujeres respecto a los hombres, debido a una sociedad que, históricamente, mantiene un sistema de relaciones de género donde destaca la superioridad de los hombres sobre las mujeres y asigna diferentes atributos, roles y espacios en función del sexo (Instituto Andaluz de la Mujer s. f.; Ministerio de Sanidad, Servicios Sociales e Igualdad 2012).

Tras sufrir un proceso de violencia, las mujeres acuden a los servicios de urgencia como punto de referencia, tanto para recibir la atención sanitaria necesaria como derivados del proceso legal que requiere un parte de lesiones. En este proceso, el servicio de urgencias se convierte pues en el principal detector de la violencia de género, puesto que es un dispositivo al alcance de las mujeres maltratadas.

\section{Violencia de género}

La definición formulada en el Convenio de Estambul entiende violencia contra las mujeres como:

(BOE N. ${ }^{\circ} 137$ 2014) Una violación de los derechos humanos y una forma de discriminación contra las mujeres, y designará todos los actos de violencia basados en el género que implican o pueden implicar para las mujeres daños o sufrimientos de naturaleza física, sexual, psicológica o económica, incluidas 
las amenazas de realizar dichos actos, la coacción o la privación arbitraria de libertad, en la vida pública o privada.

Tipos de violencia de género

Dentro del concepto violencia de género se pueden distinguir principalmente tres tipos de violencia:

- La violencia física, que incluye cualquier acto no accidental que implique el uso deliberado de la fuerza contra el cuerpo de la mujer, utilizando o no instrumentos, que provoquen o puedan provocar lesión, daño o dolor afectando a su integridad física (Ministerio de Sanidad, Servicios Sociales e Igualdad 2012; Suárez Ramos, Borrás Pruneda, Frías Oncina, Llamas Martínez y Vicuete Rebollo 2015).

- La violencia psicológica, que incluye toda conducta verbal o no verbal, intencionada y prolongada en el tiempo, que atenta contra la integridad psíquica y emocional de la mujer y contra su dignidad, produciendo dependencia, aislamiento y temor (Ministerio de Sanidad, Servicios Sociales e Igualdad 2012; Suárez Ramos, Borrás Pruneda, Frías Oncina, Llamas Martínez y Vicuete Rebollo 2015).

- La violencia sexual, que incluye todo acto de naturaleza sexual forzada por el agresor o no consentida por la mujer, donde se impone un comportamiento sexual contra la voluntad de la mujer a través de la coacción, la intimidación, la amenaza o la fuerza, independientemente de que sea por parte de la pareja o de otras personas (Ministerio de Sanidad, Servicios Sociales e Igualdad 2012; Fundación Avón s. f.).

\section{Consecuencias en la salud de las mujeres}

La Organización Mundial de la Salud (OMS) identifica la violencia de género como un factor muy importante en el deterioro de la salud de las mujeres, puesto que los tipos de violencia mencionados anteriormente suponen pérdidas en la esfera biológica, psicológica y social. Este tipo de violencia presenta repercusiones en la salud física, emocional, sexual, reproductiva y social, que permanecen aún cuando la violencia ha cesado (Ministerio de Sanidad, Servicios Sociales e Igualdad 2012).

\section{Epidemiología}

La OMS, en un análisis mundial llevado a cabo en el 2013, observó que el $35 \%$ de las mujeres había sido o será objeto de violencia física o sexual, ya sea dentro o fuera de la pareja (Organización Mundial de la Salud 2017).

En el ámbito nacional, en el año 2018, a fecha de 19 de diciembre, se produjeron 47 víctimas mortales de la violencia de género (Ministerio de la Presidencia, Relaciones con las Cortes e Igualdad 2018). A fecha de 15 de febrero de 2019 se han producido 9 víctimas mortales desde el inicio de año (Ministerio de la Presidencia, Relaciones con las Cortes e Igualdad 2019). 


\section{Urgencias}

El servicio de urgencias es un dispositivo de fácil acceso donde pueden acudir las mujeres que sufren cualquier tipo de maltrato ante la necesidad de atención urgente, por lo que estos servicios tienen una posición privilegiada para detectar e intervenir en situaciones de violencia de género. Es poco frecuente que el personal sanitario sospeche o haga preguntas a las mujeres que atiende sobre si existe violencia por parte de su pareja $\mathrm{y}$, como consecuencia, se infravalora la prevalencia de este fenómeno.

La actuación sanitaria en una mujer víctima de la violencia de género que acude a un servicio de urgencias debe ir encaminada, en primer lugar, a la atención de las lesiones y síntomas, valorando siempre su seguridad y el riesgo en el que se encuentra; en segundo lugar, facilitar la salida de la violencia a la víctima (Alarte Garví 2015).

\section{Justificación}

La elección de este tema se ha visto motivada por una problemática social que, en pleno siglo XXI, sigue acabando con la vida de las mujeres por el mero hecho de ser mujeres.

La lucha contra la violencia de género desde el ámbito sanitario debe comenzar por una correcta formación de los profesionales. Se deben proporcionar herramientas adecuadas para poder realizar las detecciones de forma precoz y es necesario unificar la información diseminada en diferentes publicaciones en documentos que permitan un fácil entendimiento.

\section{Objetivos}

General

- Dar a conocer los diferentes métodos disponibles para detectar la violencia de género en el servicio de urgencias.

\section{Específicos}

- Mostrar los métodos de detección y derivación empleados en el servicio de urgencias.

- Conocer los factores de los profesionales sanitarios que influyen en la detección de la violencia de género.

- Definir la figura de sexual assault nurse examiner (SANE).

- Detectar la existencia de colectivos vulnerables dentro del núcleo de posibles victimas.

\section{Metodología}

En este estudio se realizó una revisión integradora de carácter descriptivo de los artículos originales relacionados con los métodos de detección de la violencia de género en los servicios de urgencias. 
Se llevó a cabo una búsqueda bibliográfica en distintas bases de datos: Medline/PubMed, Cinahl, Biblioteca Virtual de la Salud (BVS) y Biblioteca Cochrane Plus.

La estrategia de búsqueda se realizó a partir de la formulación de una pregunta clínica de estructura PIO, quedando formulada de la siguiente manera: herramientas de detección para identificar casos de violencia de género en mujeres que acuden al servicio de urgencias y son atendidas por los profesionales de enfermería.

Los tesauros utilizados para llevar a cabo la búsqueda han sido mediante los Descriptores de Ciencias de la Salud (DeCS), empleados en la base de datos de la Biblioteca Virtual de la Salud; y los Medical Subject Headings (MeSH), utilizados en las bases de datos de PubMed, Cinahl y Cochrane.

Se aplicó la misma estrategia de búsqueda para las diferentes bases de datos durante el proceso de recogida documental, mediante el uso y la combinación de los descriptores $\mathrm{MeSH}$ y DeCS con los operadores booleanos $O R$ y $A N D$, facilitando la recuperación y obtención de un mayor número de resultados.

Tras realizar un cribado de los artículos recuperados, se evaluó la calidad de los artículos seleccionados mediante una lectura crítica con el uso de herramientas proporcionadas por la organización CASPe (Critical Appraisal Skills Programme español).

\section{Resultados}

Se identificaron un total de 125 artículos $(\mathrm{N})$ mediante las estrategias de búsqueda mencionadas en el apartado anterior. Tras la lectura del título y resumen, se recuperaron un total de $99,2 \%(n=124)$ artículos, de los cuales se incluyeron $8,87 \%$ $(n=11)$ artículos que cumplían con los criterios de inclusión del estudio.

En referencia a los artículos incluidos en el estudio, 8,87\% ( $n=11)$, un 36,36\% ( $n=4)$ corresponden a la base de datos de PubMed, un $9,09 \%(n=1)$ a la Biblioteca Cochrane Plus, un $0 \%(n=0)$ a la Biblioteca Virtual de la Salud y un $54,54 \%(n=6)$ corresponde a la base de datos de Cinahl.

Se descartaron un total de $114(91,2 \%)$ artículos. El principal motivo de exclusión fue no estar relacionado con el tema a tratar, con un $96,49 \%(n=110)$.

\section{Discusión y conclusiones}

\section{Métodos de detección y derivación}

Wolff, Cantos, Zun y Taylor (2017) llevaron a cabo un estudio en el cual se concluyó que la detección directa en servicios sanitarios puede ser una forma eficaz de identificar a las mujeres víctimas de la violencia de género. Concretamente, utilizar un cuestionario directo como puede ser Partner Violence Scale (PVS) puede mejorar las tasas de detección. PVS es un cuestionario con cuatro elementos principales que evalúa la victimización por violencia física y la percepción de seguridad de las víctimas. Asimismo, exponen que realizar el cuestionario a todas las mujeres aumenta el conocimiento de la prevalencia y reduce sus sentimientos de aislamiento. Además, añaden que proporcionar una información adecuada a mujeres en las que se detecta 
violencia de género favorece que estas se pongan en contacto con los recursos y la ayuda necesarios.

En la misma línea de investigación, Ahmad, Ali, Rehman, Talpur y Dhingra (2017) indican que la detección sistemática o universal produce tasas más altas de identificación de casos de violencia de género. Asimismo, informan de que existen varios métodos de detección como los cuestionarios realizados por ordenador, escritos, cuestionarios en audio y verbales. La efectividad de estos depende del contexto en el que se realicen, la confianza y la comodidad entre la paciente y el profesional y la disposición y el estado de la persona.

Por otro lado, en la revisión bibliográfica llevada a cabo por Sorenson, Joshi y Sivitz (2014) se pone de manifiesto la importancia de añadir un apartado en las encuestas de violencia de género sobre el estrangulamiento o ahogamiento, pues la estrangulación previa es un factor de pronóstico del homicidio de mujeres víctimas de la violencia de género.

Couden Hernandez, Reibling, Maddux y Kahn (2015) en su estudio muestran la escala Conflict Tactics Scale, cuyos ítems evalúan la exposición personal al abuso infantil y la violencia de género, incluyendo el daño físico y las relaciones sexuales forzadas. Asimismo, hacen referencia al NorVold Abuse Questionnaire donde se evalúa la exposición a violencia física, sexual o emocional y se proporcionan criterios específicos en tres niveles: leve, moderado o severo.

Del mismo modo, Ritchie, Nelson, Wills y Jones (2014) exponen en su estudio que, para realizar una implantación exitosa de la intervención sobre la violencia de género, hace falta un cambio de actitud y comportamiento en el personal sanitario, donde también se ven involucrados un conjunto de sistemas que incluyen la gestión, la colaboración interinstitucional y la formación obligatoria del personal, entre otros.

En la atención a una paciente que ha sufrido violencia de genero, la documentación es fundamental para apoyar una intervención efectiva y puede ser utilizada para solicitar órdenes de protección, por lo que debe detallar la historia obtenida, registrar la ubicación y la extensión de las lesiones y la intervención proporcionada. Además, desarrolla una herramienta de diecisiete ítems para la evaluación de las historias clínicas de mujeres víctimas de abusos sexuales y de pareja.

En relación con la notificación de los casos detectados, Oliveira Friestino (2016) pone de manifiesto que la notificación obligatoria de los casos de violencia infantil y doméstica es un buen instrumento para el seguimiento y proporciona información a los servicios de salud, de forma que se pueda integrar la atención y las estrategias para abordar el problema.

Por último, Hewitt (2015) en su estudio hace referencia a las lesiones más comunes en las mujeres víctimas de la violencia de género. Además, añade que es muy importante documentar los hechos ocurridos, donde se incluye el examen físico y la documentación escrita, que deben ser lo más específicos posible. Por otro lado, se propone el Partner Violence Screen (PVS), una herramienta de evaluación de tres preguntas, donde una respuesta afirmativa en cualquiera de las tres preguntas se considera positivo en violencia de género. Asimismo, defiende la evaluación universal y promueve la presencia de pósteres, folletos y cuestionarios de rutina colocados estratégicamente para aumentar la conciencia y la detección de la violencia de género. 


\section{Factores que influyen en la detección}

En la revisión bibliográfica realizada por Ahmad, Ali, Rehman, Talpur y Dhingra (2017) se hace mención a una serie de factores que afectan a la capacidad de los profesionales de la salud para detectar la violencia de género en los servicios de urgencias. Entre ellos se encuentran los siguientes:

- En cuanto a los factores relacionados con el profesional de la salud, podemos encontrar la falta de atención y empatía, así como la falta de formación.

- Los factores relacionados con la estructura organizativa son la falta de privacidad, la falta de servicios sociales fuera de horario y la falta de tiempo.

- Entre los factores relacionados con la paciente, se encuentran la falta de disposición para compartir o abordar el tema, la falta de confianza con el profesional que le atiende, la sensación de vergüenza y el temor al maltratador o a la pérdida de sus hijos.

\section{Sexual Assault Nurse Examiner (SANE)}

Thomas, Scott Tilley y Esquibel (2015) en su estudio indican que es apropiado la presencia de personal de enfermería examinador del asalto sexual (SANE) que haya sido formado específicamente para el cuidado y la atención a las víctimas de agresión sexual, así como para satisfacer sus necesidades. Por otro lado, este estudio menciona las lesiones físicas más comunes que presenta una mujer tras una agresión sexual y recomienda los exámenes colposcópicos para realizar un examen físico adecuado, ya que son los más indicados para visualizar las abrasiones genitales y hematomas que no pueden verse a simple vista.

En la misma línea de investigación se encuentra el estudio realizado por Capri Delgadillo (2017) en el que se destaca también la importancia de la presencia de SANE en el servicio de urgencias. Además, se proporcionan indicaciones para el personal de enfermería que trabaja en el servicio de urgencias, puesto que no siempre está presente la figura de SANE. En primer lugar, se debe proporcionar una habitación privada donde un trabajador social esté con la paciente, también puede estar un acompañante si ella lo desea. El personal debe ser amable y ofrecer apoyo a lo largo de la atención. Posteriormente se debe realizar el examen físico junto con el médico y registrarlo en la historia clínica de la paciente. En cuanto a la obtención del examen forense, se debe realizar tras el consentimiento. Por último, se realizan las pruebas y profilaxis de enfermedades de transmisión sexual (ITS) y se proporcionan las medidas adecuadas para la prevención del embarazo, según la situación de cada mujer.

Del mismo modo, la revisión bibliográfica realizada por Adams y Hulton (2016) habla sobre SANE como un miembro vital de los equipos de respuesta del asalto sexual (SART), cuyos objetivos son proporcionar una correcta atención a la víctima y educar en prevención.

\section{Colectivos vulnerables}

Austin, Lews y Washington (2014) realizaron una revisión bibliográfica donde concluyeron que las mujeres con algún tipo de discapacidad tienen un riesgo incrementado de sufrir abuso físico, emocional, sexual y verbal por parte de su pareja. El abuso sexual y las relaciones sexuales no deseadas es la violencia más común entre mujeres con discapacidad o deficiencias cognitivas. Además, las mujeres con 
discapacidad embarazadas tienen un riesgo mayor de sufrir abuso físico antes y durante el embarazo. Esto se conoce como abuso especifico de la discapacidad.

Además, plantean una serie de factores que se deben tener en cuenta a la hora de identificar la violencia de género en personas con discapacidad, entre ellos se encuentran la dependencia del cuidado de su pareja, la existencia de hijos en común, la dependencia económica de la mujer y la presencia de limitaciones cognitivas.

Por último, proponen el cuestionario Abuse Assessment Screen-D (AAS-D) como una herramienta específica que evalúa el riesgo potencial o peligro futuro de mujeres con discapacidad que dan positivo en cualquier tipo de abuso.

\section{Conclusiones}

- Es importante la realización de un cuestionario universal, ya que no solo aumenta las tasas de detección, sino que incrementa la concienciación de la violencia de género.

- Se deben tener en cuenta los factores que afectan la detección de la violencia de género para actuar sobre estos y mejorar las tasas de detección.

- Se recomienda la presencia de personal formado en la detección del abuso sexual en los servicios de urgencias.

- El personal de enfermería debe conocer los procedimientos a llevar a cabo y la correcta recopilación de datos ante un abuso sexual.

- Se debe prestar especial atención a aquellas mujeres con algún tipo de discapacidad.

\section{Referencias bibliográficas}

Adams, Phyllis y Linda Hulton. 2016. "The Sexual Assault Nurse Examiner's Interactions Within the Sexual Assault Response Team». Advanced Emergency Nursing Journal, 38(3): 213-227. https://doi.org/10.1097/TME.0000000000000112

Ahmad, Irfan, Parveen Azam Ali, Salma Rehman, Ashfaque Talpur y Katie Dhingra. 2017. "Intimate partner violence screening in emergency department: a rapid review of the literature». Journal of Clinical Nursing, 26(21-22): 3271-3285. https://doi.org/10.1111/jocn.13706

Alarte Garví, Jose Manuel. 2015. Impacto Médico-Legal de las asistencias por Violencia Doméstica en un Servicio de Urgencias Hospitalario. Murcia: Universidad de Murcia.

Austin, Brooke, Jennifer Lews y Robin Washington. 2014. «Women with Disabilities and Interpersonal Violence: A Literature Review». Journal of the National Society of Allied Health, 11(1): 42-54. Recuperado de: https://search.ebscohost.com/login.aspx?direct=true\&db=ccm\&AN=128026513 \&site=ehost-live

Boletín Oficial del Estado n. ${ }^{\circ}$ 137. 2014. Instrumento de ratificación del Convenio del Consejo de Europa sobre prevención y lucha contra la violencia contra la mujer y la violencia doméstica, hecho en Estambul el 11 de mayo de 2011. Diario Oficial Boletín Oficial del Estado, 42949. Recuperado de http://www.boe.es

Capri Delgadillo, Destiny 2017. «When There is No Sexual Assault Nurse Examiner: Emergency Nursing Care for Female Adult Sexual Assault Patients». Journal of Emergency Nursing, 43(4): 308-315. https://doi.org/10.1016/j.jen.2016.11.006 
Couden Hernandez, Barbara, Ellen T. Reibling, Charles Maddux y Michael Kahn. 2015. «Intimate Partner Violence Experienced by Physicians: A Review». Journal of Women's Health, 25(3): 311-320. https://doi.org/10.1089/jwh.2015.5216

Fundación Avón. s. f. Tipos de Violencia de género - Fundación Avon. Recuperado de: https://www.fundacionavon.org.ar/tipos-de-violencia-domestica/ [Consultado el 8 de enero de 2019].

Hewitt, Lisa N. 2015. "Intimate Partner Violence. The Role of Nurses in Protection of Patients». Critical Care Nursing Clinics of North America, 27(2): 271-275. https://doi.org/10.1016/j.cnc.2015.02.004

Instituto Andaluz de la Mujer. s.f. Violencia de género. Recuperado de: http://www.juntadeandalucia.es/iam/index.php/areas-tematicas/violencia-degenero [Consultado el 8 de enero de 2019].

Ministerio de la Presidencia, Relaciones con las Cortes e Igualdad 2019. Mujeres Víctimas Mortales por Violencia de Género en España a manos de sus parejas o exparejas. Recuperado de:

http://www.violenciagenero.igualdad.mpr.gob.es/violenciaEnCifras/victimasMort ales/fichaMujeres/pdf/VMortales_2019_06_05.pdf

Ministerio de la Presidencia, Relaciones con las Cortes e Igualdad. 2018. Mujeres Víctimas Mortales por Violencia de Género en España a manos de sus parejas o exparejas. Recuperado de:

http://www.violenciagenero.igualdad.mpr.gob.es/violenciaEnCifras/

Ministerio de Sanidad, Servicios Sociales e Igualdad. 2012. Protocolo común para la actuación sanitaria ante la Violencia de Género. 2012. Recuperado de: http://www.violenciagenero.igualdad.mpr.gob.es/en/profesionales/nvestigacion/ sanitario/docs/PSanitarioVG2012.pdf

Oliveira Friestino, Jane Kelly 2016. «Actions for Coping Domestic Violence Against Children and Teenagers: a Reflective Analysis». Journal of Nursing UFPE / Revista de Enfermagem UFPE, 10: 341-346. https://doi.org/10.5205/reuol.790180479-1-SP.1001sup201620

Organización Mundial de la Salud. 2017. Violencia contra la mujer. Recuperado de: https://www.who.int/es/news-room/fact-sheets/detail/violence-against-women [Consultado el 8 de enero de 2019].

Ritchie, Miranda, Katherine Nelson, Russell Wills y Lisa Jones. 2014. «Development of an Audit Tool to Evaluate the Documentation of Partner Abuse Assessments within a Provincial Emergency Department: An Exploratory Study». Journal of Family Violence, 29(2): 215-221. https://doi.org/10.1007/s10896-013-9566-1

Sorenson, Susan B., Manisha Joshi y Elizabeth Sivitz. 2014. «A systematic review of the epidemiology of nonfatal strangulation, a human rights and health concern». American Journal of Public Health, 104(11): e54-e61.

https://doi.org/10.2105/AJPH.2014.302191

Suárez Ramos, Amalia, Syra Borrás Pruneda, Ifigenia Frías Oncina, M. ${ }^{a}$ Victoria Llamas Martínez y Elisa Vicuete Rebollo. 2015. Protocolo andaluz para la actuación sanitaria ante la violencia de género. Recuperado de: https://www.juntadeandalucia.es/export/drupaljda/salud_5af95874e02ad_protoc olo_violencia_genero.pdf

Thomas, Laura, Donna Scott Tilley y Karen Esquibel. 2015. «Sexual Assault: Where Are Mid-Life Women in the Research?». Perspectives in Psychiatric Care, 51(2): 86-97. https://doi.org/10.1111/ppc.12067

Wolff, Jennifer, Arthur Cantos, Leslie Zun y Ashley Taylor. 2017. «Enhanced Versus Basic Referral for Intimate Partner Violence in an Urban Emergency Department Setting». Journal of Emergency Medicine, 53(5): 771-777. https://doi.org/10.1016/j.jemermed.2017.06.044

Xunta de Galicia. s.f. ¿Qué es la violencia de género? Recuperado de: http://igualdade.xunta.gal/es/content/que-es-la-violencia-de-genero [Consultado el 8 de enero de 2019]. 